\title{
Influence of Closed Chain Vs Open Chain Exercises in Patients with Chronic Ankle Arthritis
}

\author{
Tapan Haresh Shah', Siva Kumar Raman², Kunal Chandrakant Shah ${ }^{3}$
}

\begin{abstract}
${ }^{1}$ B.PT, M.PT (Musculoskeletal \& Sports) Vikas College of Physiotherapy, Mangalore, India.
${ }^{2}$ B.PT, M.PT Professor Vikas College Of Physiotherapy, Mangalore, India.

${ }^{3}$ MS Ortho. Sancheti Institute for Orthopedics and Rehabilitation, Pune, India.

Institute at which research was conducted: Vikas College of Physiotherapy, Mangalore, India. University Affiliation of Thesis: Rajiv Gandhi university of Health Sciences.

Year of Acceptance: 2012
\end{abstract}

\section{Address of Correspondence}

Dr Tapan Shah

E/41 Mukand Society, vrindavan, L.B.SMarg, Ghatkopar (West), Mumbai 400086.

Maharashtra, India.

,E mail: tapanshah41@gmail.com
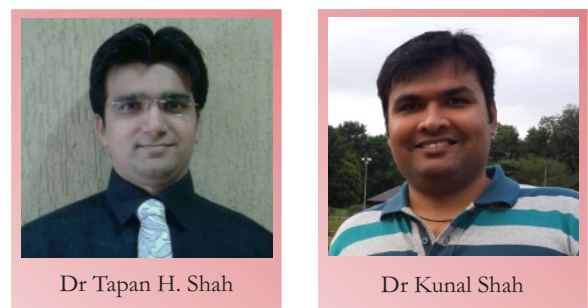

Abstract: Background: Chronic ankle arthritis is often managed by exercises. But there is no adequate literature about the superiority of CKC exercise over OKC exercise in reducing pain, improving ankle ROM and increasing single leg stance duration in ankle arthritis. Hence this study is attempted to determine the efficacy of CKC exercise over OKC exercise in chronic ankle arthritis.

Materials and methods: The study included $30(n=30)$ subjects who ranged in age from 40 to 60 years of both genders with chronic ankle arthritis. Subjects were assigned randomly to one of two groups, each group consisting of 15 subjects. Group I was instructed to perform CKC ankle exercise. Group II was instructed to perform CKC ankle exercise. Before the beginning of the trial and at end of six weeks pain using VAS, ankle JROM using universal goniometer and single leg stance duration were measured.

Result: The initial evaluation showed that, there is no significant difference between the two groups for all the variables measured. The post-test evaluation showed a statistically significant $(P<0.05)$ increase in the pain reduction, ankle JROM and single leg stance duration within the groups.

Conclusion: This study led to the conclusion that CKC ankle exercise and OKC ankle exercise are equally effective in pain reduction, and improving ankle JROM and single leg stance duration in chronic ankle arthritis.

Keywords: Ankle arthritis, Open kinetic chain exercise, Closed kinetic chain exercise, Ankle rehabilitation.

\section{THESIS SUMMARY}

\section{Introduction}

Primary osteoarthritis in the ankle is rare, and that secondary osteoarthritis that follows rotational ankle fractures or recurrent ligamentous instability is much more common. The primary aim of treatment is to provide pain relief. This may be attempted through the use of offloading strategies such as assistive devices. A single point cane can decrease vertical loading by $11-25 \%$. Total contact casts, patellar tendon bearing braces, and removable walking boots have been shown to offload the foot. However, they induce asymmetrical loading, and are often accompanied by poor patient compliance. Application of heat modalities and exercise are the mainstay of treatment for increasing muscle strength, JROM and function. Though exercise is the mainstay in the management of chronic ankle arthritis, there is no consensus regarding the efficacy of closed chain exercise(CKC) ankle exercise and open chain exercise(OKC) exercise. Also majority of literature pertains to knee joint and there is a dearth of studies on ankle joint CKC exercise. Hence we attempt to 
determine the efficacy of CKC ankle exercise in the management of chronic ankle arthritis.

\section{Materials and methods:}

We performed a prospective randomised control trial and included 30 $(\mathrm{n}=30)$ subjects who ranged in age from 40 to 60 years of both genders with chronic ankle arthritis. All patients were symptomatic for more than 3 months. The exclusion criteria were History of trauma to the lower limbs history of lower limb surgery, radiating pain from lumbosacral disorders, generalized inflammatory disorders associated with the diagnosis of rheumatoid arthritis, ankylosing spondylitis, Reiter's disease, gout, or lupus, use of pain control (analgesics, non-steroidal anti-inflammatory drugs (NSAIDs) and steroids at the time of recruitment.

Subjects were assigned randomly to one of two groups, each group consisting of 15 subjects. Group I was instructed to perform CKC ankle exercise. Group II was instructed to perform $\mathrm{CKC}$ ankle exercise. Before the beginning of the trial and at end of six weeks pain using VAS, ankle JROM using universal goniometer and single leg stance duration were measured. The data collected by VAS and single leg stance was analyzed using non-parametric tests as the data were ordinal in nature. The intra group pre and post-test data was analyzed using Wilcoxon signed rank test, while the post-test inter group data was be analyzed Mannwhitney $U$ test. The data collected by goniometric measurement of the ankle joint was analyzed using paired $t$ test for intra group and unpaired t test for inter group.

\section{Results:}

The initial evaluation showed that, there is no significant difference $(\mathrm{P}>0.05)$ between the two groups for all the variables measured. The post-test evaluation showed a statistically significant $(\mathrm{P}<0.05)$ increase in the pain reduction, ankle JROM and single leg stance duration within the groups. A post-test comparison between the groups showed that there is no statistically significant $(\mathrm{P}>0.05)$ between the groups.

\section{Conclusion:}

This study led to the conclusion that CKC ankle exercise and OKC ankle exercise are equally effective in pain reduction, and improving ankle JROM and single leg stance duration in chronic ankle arthritis. However a long term intervention and regular follow up over an extended period of time are necessary to interpret the results reliably.

\section{Key Words:}

Ankle arthritis, Open kinetic chain exercise, Closed kinetic chain exercise, Ankle rehabilitation.

\section{Bibliography}

1.Praemer AP, Furner S, Rice DP. Musculoskeletal Conditions in the United States. Rosemont, Illinois: American Academy of Orthopaedic Surgeons; 1999. p. 182.

2.Cushnaghan J, Dieppe P. Study of 500 patients with limb joint osteoarthritis. I. Analysis by age, sex, and distribution of symptomatic joint sites. Ann Rheum Dis. 1991 Jan;50(1):8-13.

3.Demetriades L, Strauss E, Gallina J. Osteoarthritis of the Ankle Joint. Clin Orthop. 1998;349:28-42.

4. Wyss $C$, Zollinger $H$. The causes of subsequent arthrodesis of the ankle joint. Acta Orthop Belg. 1991;57(suppl 1):22-27.

5.Taga I, Shino K, Inoue M, Nakata K, Maeda A. Articular cartilage lesions in ankles with lateral ligament injury: An arthroscopic study. Am J Sports Med. 1993;21:120-126.

6.Inokuchi S, Ogawa K, Usami N, Hashimoto T. Long-term follow up of talus fractures. Orthopaedics. 1996;19:477-481.

7.Martin RL, Stewart GW, Conti SF. Posttraumatic ankle arthritis: an update on conservative and surgical management. J Orthop Sports Phys Ther 2007; 37:253-259.

8.Aragaki DR, Nasmyth MC, Schultz SC, et al. Immediate effects of contralateral and ipsilateral cane use on normal adult gait. PMR 2009; 1:208-213.

9.DiLiberto FE, Baumhauer JF, Wilding GE, et al. Alterations in plantar pressure with different walking boot designs. Foot Ankle Int 2007; 28:55-60.

10.Keefer M, King J, Powell D, et al. Effects of modified short-leg walkers on ground reaction force characteristics. Clin Biomech (Bristol, Avon) 2008; 23:1172-1177.

11. Steine HA, Brosky T, Reinking MF, Nyland J, Mason MB: A Comparison of closed kinetic chain and isokinetic joint isolation exercise in patients with patellofemoral dysfunction. JOrthop Sports Phys Ther 24(3): 136-141, 1996.

12. Brody LT., Thein JM.: Non operative treatmrnt for patellofemoral pain. J Orthop sports phys ther 28(5): 336/341, 1998 .

13. Witvrouw E, Lysens R, Bellemans J, Peers K, Vanderstraeten G: Open versus closed kinetic chain exercises for patellofemoral pain. Am J Sports Med 28(5):687, 2000.

14.Blackburn JR, Morrissey MC: The relationship between open and closed kinetic chain strength of the lower limb and jumping performance. J Orthop Sports Phys ther 27(6); $434,1998$.

15.Morrissey MC, Drechsler WI, Morrissey D, Knight PR, Armstrong PW, McAuliffe TB: Effects of distally fixated versus nondistally fixated leg extensor resistance training on knee pain in the early period after anterior cruciate ligament reconstruction. Phys. Ther. 2002; 82:35-43.

16. Mikkelsen C, Werner S, Eriksson E: Closed kinetic chain alone compared to combined open and closed kinetic chain exercises for quadriceps strengthening after anterior cruciate ligament reconstruction with respect to return to sports: a prospective matched follow-up study. Knee Surg Sports Traumatol Arthrosc 8(6):337-342,2000.
Conflict of Interest: Nil

Source of Support: None
Full Thesis and Master Chart available on www.journalmedicalthesis.com

How to Cite this Article:

Shah TH, Raman SK, Shah KC. Influence of Closed Chain Vs Open Chain Exercises in Patients with Chronic Ankle Arthritis. Journal Medical Thesis 2013 July-Sep; 1(1):12-13 